\title{
Pre-hospital trauma care in Switzerland and Germany: do they speak the same language?
}

\author{
Kai Oliver Jensen ${ }^{1}$ (1) - Michel Paul Johan Teuben ${ }^{1} \cdot$ Rolf Lefering $^{2} \cdot$ Sascha Halvachizadeh $^{1} \cdot$ Ladislav Mica $^{1}$. \\ Hans-Peter Simmen ${ }^{1} \cdot$ Roman Pfeifer $^{1} \cdot$ Hans-Christoph Pape ${ }^{1} \cdot$ Kai Sprengel $^{1} \cdot$ The TraumaRegister DGU $^{2}$
}

Received: 13 November 2019 / Accepted: 14 January 2020 / Published online: 29 January 2020

○) Springer-Verlag GmbH Germany, part of Springer Nature 2020

\begin{abstract}
Purpose Swiss and German (pre-)hospital systems, distribution and organization of trauma centres differ from each other. It is unclear if outcome in trauma patients differs as well. Therefore, this study aims to determine differences in characteristics, therapy and outcome of trauma patients between both German-speaking countries.

Methods The TraumaRegister DGU ${ }^{\circledR}$ (TR-DGU) was used. Patients with Injury Severity Score $\geq 9$ admitted to a level 1 trauma centre between 01/2009 and 12/2017 were included if they required ICU care or died. Trauma pattern, pre-hospital procedures and outcome were compared between Swiss $(\mathrm{CH}, n=4768)$ and German (DE, $n=66,908)$ groups.

Results Swiss patients were older than German patients (53 vs. 50 years). ISS did not differ between groups (CH 23.8 vs. DE 23.0 points). There were more low falls $<3 \mathrm{~m} \mathrm{(34 \%} \mathrm{vs.} 21 \%)$ at the expense of less traffic accidents (37\% vs. $52 \%)$ in the Swiss population. In Switzerland 30\% of allocations were done without physician involvement, whereas this occurred in $4 \%$ of German cases. Despite a comparable number of patients with a GCS $\leq 8$ ( $\mathrm{CH} 29.6 \%$; DE 26.4\%), differences in prehospital intubation rates occurred (CH $31 \%$ vs. DE 40\%). Severe traumatic brain injuries were diagnosed most frequently in Switzerland (CH 62\% vs. DE 49\%). Admission vital signs were similar, and standardized mortality ratios were close to one in both countries.

Conclusion This study demonstrates that patients' age, trauma patterns and pre-hospital care differ between Germany and Switzerland. However, adjusted mortality was almost similar. Further benchmarking studies are indicated to optimize trauma care in both German-speaking countries.
\end{abstract}

Keywords International comparison $\cdot$ Switzerland $\cdot$ Germany $\cdot$ Trauma systems

\section{Abbreviations}

EMS Emergency medical service

HEMS Helicopter emergency medical service

DE German cohort

Kai Oliver Jensen and Michel Paul Johan Teuben contributed equally.

The TraumaRegister DGU: Committee on Emergency Medicine, Intensive Care and Trauma Management (Sektion NIS) of the German Trauma Society (DGU).

Kai Oliver Jensen

kaioliver.jensen@usz.ch

1 Division of Trauma Surgery, Department of Trauma, University Hospital Zurich, University of Zurich, Raemistrasse 100, 8091 Zurich, Switzerland

2 Institute for Research in Operative Medicine (IFOM), University Witten/Herdecke, Cologne, Germany

$\begin{array}{ll}\text { CH } & \text { Swiss cohort } \\ \text { TR-DGU } & \text { TraumaRegister DGU } \\ \text { pRBC } & \text { Packed red blood cells } \\ \text { SMR } & \text { Standardized mortality ratio }\end{array}$

\section{Introduction}

Implementation of standardized guidelines for pre-hospital care as well as ongoing optimization of trauma system/ network organization has resulted in improved outcome in trauma patients worldwide [1-4], although pre-hospital care systems, guidelines and organization of local trauma networks differ between countries. This also applies to two mainly German-speaking countries: Germany (DE) and Switzerland $(\mathrm{CH})[5,6]$. Documented differences have both historical and geographical backgrounds. Geographical wise, total driving distance in Germany (709 billion vehicle $\mathrm{km}$ ) is 
about ten times higher compared with Switzerland, whereas the Swiss landscape is characterized by ten times more hills and mountains [7]. Additionally, on Swiss freeways speed limits are mainly set at $120 \mathrm{~km} / \mathrm{h}$, in Germany in general no speed limitations are defined for most freeways [8].

Furthermore, the German emergency system is known for its physician-based pre-hospital system. This includes either a ground emergency medical service (EMS) or a helicopter emergency medical service (HEMS). EMS teams are composed of a specialized ambulance crew with paramedics and mostly a medical physician to support with treating unstable patients [9]. HEMS teams always include both a paramedic and an emergency physician [10, 11]. In Switzerland, on the other hand, EMS teams are mainly composed of well-trained paramedics, rather than medical physicians depending on regional differences. In the case physicians are believed to be required on the spot, HEMS teams with a 24/7 service could be involved [12].

As almost $60 \%$ of trauma fatalities in the Western world occur prior to hospital admission, it is essential to optimize pre-hospital care [13]. This study aimed to determine differences in injury pattern, pre-hospital care and outcome of trauma patients between both German-speaking countries.

\section{Patients and methods}

The TraumaRegister DGU ${ }^{\circledR}$ (TR-DGU) of the German Trauma Society (Deutsche Gesellschaft für Unfallchirurgie, DGU) was utilized for this retrospective analysis. This registry was founded in 1993. The aim of this multi-centre database is a pseudonymized and standardized documentation of severely injured patients. Data are collected prospectively in four consecutive time phases from the site of the accident until discharge from hospital: (A) pre-hospital phase, (B) emergency room and initial surgery, $(\mathrm{C})$ intensive care unit and (D) discharge. The documentation includes detailed information on demographics, injury pattern, comorbidities, pre- and in-hospital management, course on intensive care unit, relevant laboratory findings including data on transfusion and outcome of each individual. The inclusion criteria are admission to hospital via emergency room with subsequent ICU/ICM care, or hospital arrival with vital signs and death before admission to ICU. The infrastructure for documentation, data management and data analysis is provided by AUC-Academy for Trauma Surgery (AUC-Akademie der Unfallchirurgie $\mathrm{GmbH}$ ), a company affiliated to the German Trauma Society. The scientific leadership is provided by the Committee on Emergency Medicine, Intensive Care and Trauma Management (Section NIS) of the German Trauma Society. The participating hospitals submit their data pseudonymized into a central database via a Web-based application. Scientific data analysis is approved according to a peer review procedure laid down in the publication guideline of TR-DGU. The participating hospitals are primarily located in Germany (90\%), but a rising number of hospitals of other countries contribute data as well (at the moment from Austria, Belgium, China, Finland, Luxembourg, Slovenia, Switzerland, The Netherlands, and the United Arab Emirates). Currently, approximately 33,000 cases from more than 650 hospitals are entered into the database per year. Participation in TraumaRegister DGU ${ }^{\circledR}$ is voluntary. For hospitals associated with TraumaNetzwerk DGU ${ }^{\circledR}$, however, the entry of at least a basic data set is obligatory for reasons of quality assurance. A detailed description of the structure and quality control of the registry is available online: https://www. traumaregister-dgu.de.

For the purpose of this study, patients admitted to Swiss $(\mathrm{CH})$ or German (DE) trauma centres in the years 2009-2017 were included if they had Injury Severity Score (ISS) $\geq 9$ with subsequent ICU/IMC care or death. Patients for whom data sets were incomplete were excluded from review and not included in the specific sub-analysis. Only supra-regional (level 1) trauma centres were considered that used the standard data set. In total, 3 out of 12 Swiss and 88 German trauma centres participated. All participating Swiss trauma hospitals were located in German-speaking areas of Switzerland.

The present study is in line with the publication guidelines of the TraumaRegister $\mathrm{DGU}^{\circledR}$ and registered as TRDGU project ID 2014-016.

\section{Statistical analysis}

Data from Swiss $(\mathrm{CH})$ and German (DE) trauma centres were compared. Data are presented as percentages with overall number of cases, or as mean with standard deviation (SD). In case of skewed distribution of data, the median was given additionally. Due to the large number of cases in both study groups, even minor differences between the groups would become formally statistically significant. Therefore, no formal significance testing was performed. Observed and expected mortality rates were analysed in primary admitted patients (without early transfers out) using the standardized mortality ratio (SMR). Expected mortality was calculated according to RISC II prognostic score which was developed and validated with TR-DGU data [14]. A 95\% confidence interval was provided for SMRs. Statistical analysis was conducted using SPSS Statistical software for Windows (Version 22.0; IBM Corp., Armonk, NY, USA).

\section{Results}

A total of 71,676 patients were evaluated in this study. A blunt trauma mechanism was observed in over $95 \%$ of cases in both study groups. The majority of patients were males 
Table 1 Patient characteristics in both study groups

\begin{tabular}{lll}
\hline & Switzerland & Germany \\
\hline Number of patients $(n)$ & 4768 & 66,908 \\
Mean age (years) (SD) & $53.5(21.8)$ & $49.9(22.2)$ \\
Age $\geq 60$ years & $42.7 \%$ & $35.4 \%$ \\
Gender (males) & $69.8 \%$ & $71.5 \%$ \\
\hline
\end{tabular}

Table 2 Mechanisms of injury

\begin{tabular}{lcc}
\hline & $\begin{array}{l}\text { Switzerland } \\
(N=4768)\end{array}$ & $\begin{array}{l}\text { Germany } \\
(N=66,908)\end{array}$ \\
\hline Blunt trauma mechanism (\%) & 95.4 & 95.7 \\
Traffic accidents, all (\%) & 37.3 & 52.1 \\
Car (\%) & 10.0 & 21.4 \\
Motorbike (\%) & 9.5 & 12.7 \\
Bicycle (\%) & 9.0 & 9.1 \\
Pedestrian (\%) & 7.2 & 7.4 \\
Other traffic (\%) & 1.6 & 1.5 \\
Low fall $<3$ m (\%) & 34.1 & 20.9 \\
Fall from height $>3$ m (\%) & 14.9 & 17.3 \\
Violence related injury (\%) & 3.1 & 2.5 \\
Gunshot wounds (\%) & 1.7 & 0.7 \\
Stab wounds (\%) & 1.9 & 1.6 \\
Suicide (\%) & 6.2 & 5.9 \\
Other (\%) & 6.1 & 5.8 \\
\hline
\end{tabular}

(CH 69.8\% and DE 71.5\%) and the mean age of patients in Switzerland was 53.5 years with $42.7 \%$ aged 60 years or older. In the German cohort, the mean age was 49.9 years and $35.4 \%$ of patients were 60 years of age or older. The baseline characteristics of both study groups are presented in Table 1.

\section{Trauma mechanisms, medical physician involvement and out-of-hospital interventions}

In Switzerland, there were nearly twice as many low falls (CH 34\% vs. DE 21\%) compared to Germany, whereas traffic accidents were more common in German patients $(\mathrm{CH}$ $37 \%$ vs. DE $52 \%$ ). Violence-related admissions were low in both countries (3.1\% vs. 2.5$)$. The mechanisms of injury are summarized in Table 2.

As expected, in Switzerland about one-third of all allocations were done by paramedics, in contrast to $4.2 \%$ in Germany. However, the frequency of invasive pre-hospital procedures was similar in both countries (2.1 per case, from a list of 6 interventions). Overall transfer times in Switzerland and Germany were very similar. However, minor differences in duration of ground transportation were seen $(\mathrm{CH}$ 69 (mean)/63 (median) min vs. DE 63/59 min). Helicopter transportation times, however, were similar (DE/CH both: 79/75 min).

Although the rate of unconscious patients (GCS $\leq 8)$ was similar (CH 29.6\%; DE 26.4\%), pre-hospital intubation rates were much lower in the Swiss cohort compared with Germany ( $\mathrm{CH} 31.2 \%$ vs. DE $40.1 \%)$. As anticipated, a lower rate of pre-hospital chest tube placements was found in Switzerland (CH $1.6 \%$ vs. DE 4.2\%), as severe thoracic trauma was also less frequently diagnosed in comparison with Germany (CH 36.5\% vs. DE 45.5\%). Out-of-hospital interventions and pre-clinical data are displayed in Table 3.

\section{Pre-hospital hemodynamics and fluid management}

At the trauma site, hypotension defined as a systolic blood pressure $\leq 90 \mathrm{mmHg}$ occurred in $8.2 \%$ of Swiss cases and in $13.0 \%$ of German trauma patients. Traumatic cardiac arrest with cardiopulmonary resuscitation was similar in both countries (CH $3.2 \%$ vs. DE $4.0 \%$ ), as was catecholamine administration $(9.8 \%$ vs. $10.7 \%)$.

Interestingly, obvious differences were seen in prehospital fluid management between both countries. In Switzerland, $637 \mathrm{ml}$ per patient was substituted, whereas German patients received $897 \mathrm{ml}$ on average per patient. On hospital admission, rates of hypotension differed only slightly between both countries, as $8.2 \%$ of Swiss patients and $11.8 \%$ of German patients had an admission systolic blood pressure $\leq 90 \mathrm{mmHg}$. Laboratory findings (including hemoglobin levels) were similar in the two study groups. The baseline values on admission are shown in Table 4 .

\section{Injuries and severity}

Table 5 shows an overview of injuries and injury severity. Mean ISS in the Swiss group (CH) was 23.8 points and similar to the German group with 23.0 points. Swiss patients suffered more frequently from severe traumatic brain injuries (TBI) than German patients (61.9\% vs. 49.4$)$, but thoracic injuries were more prevalent in Germany (36.5\% vs. $45.5 \%)$. Isolated TBI occurred with an incidence of $27.1 \%$ $(\mathrm{CH})$ compared to $16.9 \%(\mathrm{DE})$.

\section{Outcome}

The length of stay on the intensive care unit (LOS-ICU) was shorter in Switzerland compared to the German cohort (CH 5.4 vs. DE 8.7 days). In accordance, hospital LOS in Switzerland was shorter than in Germany $(\mathrm{CH} 14.6$ vs. DE 20.0 days). The number of patients receiving packed red blood cells (pRBC) was similar in both countries (about $13 \%)$. Mass transfusion ( $\geq 10 \mathrm{pRBC} /$ patient until ICU admission) was rare in both groups (CH 1.8\%; DE 2.7\%). Overall mortality rates differed between both groups and 
Table 3 Pre-hospital procedures and allocation; primary admitted patients only (no transfers)
Table 4 Admission laboratory values, hemodynamics and transfusion requirements; primary admitted patients only (no transfers)

\begin{tabular}{|c|c|c|}
\hline & $\begin{array}{l}\text { Switzerland } \\
(N=3370)\end{array}$ & $\begin{array}{l}\text { Germany } \\
(N=56,820)\end{array}$ \\
\hline \multicolumn{3}{|l|}{ Pre-hospital procedures } \\
\hline Mean no. of pre-hospital procedures per case ( $\max .6$, see below) & 2.1 & 2.1 \\
\hline Intubation $(\%)$ & 31.2 & 40.1 \\
\hline Intubation rate in unconscious patients $(\mathrm{GCS} \leq 8)(\%)$ & 82.7 & 89.7 \\
\hline Volume administration $(\%)$ & 94.4 & 92.4 \\
\hline Pre-hospital fluid administration $(\mathrm{ml})^{\mathrm{a}}$ & $637 / 500$ & $897 / 750$ \\
\hline Analgesia/sedation $(\%)$ & 67.6 & 68.6 \\
\hline Catecholamine administration (\%) & 9.8 & 10.7 \\
\hline Chest tube placement $(\%)$ & 1.6 & 4.2 \\
\hline Cardiopulmonary resuscitation $(\%)$ & 3.2 & 4.0 \\
\hline Pre-hospital time (from accident to hospital) ${ }^{\mathrm{a}}$ & $73 / 67$ & $68 / 64$ \\
\hline \multicolumn{3}{|l|}{ Mode of transportation } \\
\hline Ground transportation (\%) & 65.7 & 65.0 \\
\hline Helicopter $(\%)$ & 34.3 & 35.0 \\
\hline Physician involved (\%) & 69.2 & 95.3 \\
\hline Paramedic only (\%) & 29.5 & 4.2 \\
\hline Private transport (\%) & 1.4 & 0.5 \\
\hline
\end{tabular}

GCS Glasgow Coma Scale score

${ }^{\mathrm{a}} \mathrm{Mean} /$ median

\begin{tabular}{lll}
\hline & $\begin{array}{l}\text { Switzerland } \\
(N=1.807)\end{array}$ & Germany $(N=22.769)$ \\
\hline Haemoglobin $(\mathrm{g} / \mathrm{l})$ & $12.4(2.4)$ & $12.6(2.4)$ \\
Platelets $\left(\times 10^{3} / \mu \mathrm{l}\right)$ & $213(74)$ & $218(81)$ \\
PTT $(\mathrm{s})$ & $31(16)$ & $31(17)$ \\
Prothrombin time/Quick value (\%) & $76(22)$ & $82(23)$ \\
Base excess (mmol/l) & $-3.3(4.8)$ & $-2.3(4.9)$ \\
International normalized ratio (INR) & 1.28 & 1.24 \\
Blood pressure $\leq 90 \mathrm{mmHg}(\%)$ at scene & 8.2 & 13.0 \\
Blood pressure $\leq 90 \mathrm{mmHg}(\%)$ on admission & 8.2 & 11.8 \\
Blood transfusion until ICU (\%) & 12.6 & 13.8 \\
Mass transfusion $(\geq 10$ units of packed RBC) $(\%)$ & 1.8 & 2.7 \\
\hline
\end{tabular}

$P T T$ partial thromboplastin time, $R B C$ red blood cell was higher in Switzerland (18.2\%) compared to $14.4 \%$ in Germany, but after adjustment according to injury severity, the standardized mortality ratio was close to one in both countries (Table 6).

\section{Discussion}

The current international trauma study reveals that:

1. Swiss trauma victims admitted to level-one trauma institutes were older, more often injured due to low falls
$(<3 \mathrm{~m})$ and less frequently injured due to traffic-related accidents than German trauma patients.

2. Despite equal injury severity and a comparable amount of patients with decreased GCS scores, medical physician involvement is ten times lower in Switzerland than in Germany. Furthermore, in-field intubation and chest tube placements were performed less frequently in Switzerland. In addition, about $30 \%$ more fluid was administered pre-hospitally in German patients compared with Swiss cases.

3. Swiss trauma patients were discharged earlier than their German counterparts and patient-specific characteristics most likely caused encountered differences in mortality 
Table 5 Diagnosed injuries and injury severity

\begin{tabular}{lll}
\hline & $\begin{array}{l}\text { Switzerland } \\
(N=3370)\end{array}$ & Germany $(N=56,820)$ \\
\hline AIS $_{\text {Head }} \geq 3(\%)$ & 61.9 & 49.4 \\
$\begin{array}{l}\text { Isolated head trauma } \\
\quad \%)\end{array}$ & 27.1 & 16.9 \\
$\quad$ Initial GCS $\leq 8(\%)^{\mathrm{a}}$ & 29.6 & 26.4 \\
AIS $_{\text {Thorax }} \geq 3(\%)$ & 36.5 & 45.5 \\
AIS $_{\text {Abdomen }} \geq 3(\%)$ & 12.6 & 12.4 \\
AIS $_{\text {Extremities }} \geq 3(\%)$ & 23.0 & 28.7 \\
Injury Severity Score & $23.8(14.1)$ & $23.0(12.5)$ \\
New ISS & $30.9(15.6)$ & $28.7(15.0)$ \\
\hline
\end{tabular}

GCS Glasgow Coma Scale score, AIS Abbreviated Injury score

${ }^{\text {a }}$ Primary admitted patients only

between both German-speaking countries, as unadjusted mortality rates differ while standardized mortality ratios were similar. However, differences in crude mortality rates may be associated with differences in pre-hospital care systems between countries as well.

Comparative assessments between pre-hospital trauma systems are an essential tool to identify processes, which have potential to be optimized. International benchmarking is considered as a feasible tool to do so $[15,16]$. The current international benchmarking evaluation performed led to the identification of substantial differences in trauma populations, subsequent pre-hospital and hospital care. Despite comparable adjusted mortality in both groups, we managed to formulate specific recommendations for both groups/ countries.

Gender and age distribution in both countries is similar compared to other international investigations on severe trauma and this underlines the external validity of the current study [17, 18]. Interestingly, road accident-related trauma admissions in Switzerland are comparable to other studies from the Western world, whereas relatively higher rates were encountered in Germany [19, 20]. This observation may partly be due to the absence of speed limits in Germany and related specific national cultural driving habits [7].

We found striking differences in pre-hospital intubation rates between both groups. Pre-hospital intubation is frequently associated with altered neurological status and more specifically a reduced initial GCS score. Even though similar proportions of cases with an initial GCS $<9$ were documented in both cohorts, intubation rates were lower in the Swiss cohort. One can assume that the paramedicbased allocation system in Switzerland leads to a lower
Table 6 A comparison of outcome in both countries

\begin{tabular}{lll}
\hline & Switzerland $(N=3370)$ & Germany $(N=56,820)$ \\
\hline Treated on ICU (\%) & 96.3 & 97.6 \\
Length of ICU stay (days) $^{\mathrm{a}}$ & $5.4 / 2$ & $8.7 / 4$ \\
Hospital length of stay (days) ${ }^{\mathrm{a}}$ & $14.6 / 12$ & $20.0 / 15$ \\
Hospital mortality (\%) & 18.2 & 14.4 \\
Early mortality/<24 h (\%) & 9.0 & 6.6 \\
Observed vs. expected mortality & & \\
No. of primary patients (no transfer in/no & 3321 & 56,088 \\
transfer out) with RISC II & & \\
Mortality (\%) & 20.6 & 14.8 \\
RISC II prognosis (\%) & 17.9 & 14.8 \\
SMR (95\% CI) & $1.15(1.07-1.23)$ & $1.00(0.98-1.02)$ \\
Discharge destination & & 46.3 \\
Home (\%) & 24.6 & 24.8 \\
Rehabilitation clinic (\%) & 38.7 & 10.9 \\
Other hospital (\%) & 15.6 & 3.6 \\
Other (\%) & 3.0 & \\
Glasgow Outcome Scale & & 2.1 \\
PVS & 1.0 & 9.8 \\
Severe disability & 24.2 & 22.7 \\
Moderate disability & 24.4 & 50.5 \\
Good recovery & 31.8 & \\
\hline
\end{tabular}

ICU intensive care unit, RISC Revised Injury Severity Classification, RISC II Revised Injury Severity Classification Version II, SMR standardized mortality ratio, $C I$ confidence interval, GOS Glasgow Outcome Scale, $P V S$ permanent vegetative state

${ }^{\mathrm{a}}$ Mean/median 
intubation rate. As success rates of pre-hospital intubation are higher in physicians compared to non-physicians, it is likely that paramedics are more reluctant to intubate patients [21]. Thereby, the findings of the current study underline the benefits of emergency physician presence (reflected by higher intubation rates in the German cohort) in the treatment of craniocerebral injuries. Increased intubation rates may partly explain enhanced outcome observed in neurotrauma patients treated by emergency physicians compared with treatment systems with lower pre-hospital emergency physician attendance $[22,23]$.

On the other hand, GCS scoring on its own is a suboptimal trigger parameter for intubation and may be too subjective. However, as no differences in confirmed severe maxillofacial injuries were seen and severe TBI incidences as well as isolated TBI rates were highest in Switzerland, on-the-spot medical physician involvement routines in both countries are an interesting topic to analyze in more detail. Indications for medical physician allocation have the potential to be optimized and might change emergency personal demands in the future.

We further found a lower rate of pre-hospital tube thoracostomies in the Swiss cohort. This might partly be influenced by relatively lower numbers of severe thoracic injuries and in-field hypovolemic patients in Switzerland compared to Germany. Another potential explanation is the fact that medical physicians were less often involved in Swiss trauma cases than in German cases. We hypothesize that paramedics are more restrained to place chest tubes and this explains low application rates in Switzerland, especially as failure rates of percutaneous thoracic interventions performed by paramedics in the pre-hospital scenario are high [24].

Given the observed variability between both cohorts regarding chest tube placements, it would be interesting to compare protocols/indications and implementation of local guidelines as well as actual habits of emergency physicians. Additionally, to gain more insight into this topic it would be interesting to study incidences of delayed chest tube placement in the emergency room in Switzerland.

Transfer times by ground transportation differed between both groups. This study demonstrated that ground transportation in the Swiss cohort took longer than in the German cohort. We feel that this is mainly a result of geographical differences between countries or distinct patient evacuation conditions. Alternatively, it is tempting to hypothesize that physician-based pre-hospital care (Germany) may enhance in-field efficiency as well.

This study demonstrated a discrepancy in pre-hospital fluid administration between both countries. In Switzerland, about $30 \%$ less fluid had been administered to trauma patients than in Germany. This may be due to differences in fluid administration habits between both countries. as Also, a study from Timm et al. demonstrated that German trauma patients received significantly more volume in the prehospital setting than Dutch trauma patients [25]. A slightly higher amount of hypovolemic and catecholamine requiring individuals was found in the German cohort. This may also partly explain the observed differences.

Interestingly, in Switzerland the percentage of patients with in-field hypovolemia did not drop during pre-hospital transport (8.2\%), using their restricted fluid administration protocols. In contrast, more liberal fluid administration protocols, as utilized in Germany, lead to a slight decrease of hypovolemic patients as they are diagnosed on the spot (13.0\%) and subsequently admitted to the hospital (11.8\%). Thereby, more liberal pre-hospital fluid administration seems to be associated with more adequate restoration of hemodynamics during the pre-hospital phase. This is, however, in contrast with the present heavily propagated principles of limited volume resuscitation and permissive hypotension in trauma [26, 27].

Furthermore, a slightly higher percentage of patients in the German population received $>10$ units of erythrocyte concentrate transfusions than the Swiss population, despite equal admission hemoglobin levels and coagulation status. This is most likely due to the larger fraction of patients with impaired hemodynamic status (reflected by $\mathrm{SBP}<90 \mathrm{mmHg}$ on admission) in the German cohort than in the Swiss group. Nevertheless, it may also suggest different transfusion habits between both countries, especially, as transfusion habits in certain Swiss-level-one trauma centers changed markedly within the last decade [28]. Swiss transfusion rates probably dropped due to the implementation of a goal-directed coagulation algorithm, and increasing utilization of tranexamic acid and coagulation factor XIII [28, 29].

As anticipated, non-physician allocation in Switzerland was almost ten times more frequent than in Germany and in line with other studies this seems to be related with fewer in-field interventions [30]. Despite this striking difference and in contrast to literature, the adjusted outcome of trauma patients treated under both conditions in the current study was comparable [31]. However, crude mortality differed between countries and it is tempting to hypothesize that this is associated with pre-hospital emergency physician involvement.

As guidelines for the treatment of severely injured patients in the Western world are fairly standardized, we believe that the outcome of care of our cohorts is representable for most well-developed countries. The validity of our findings was underlined by the fact that the encountered crude mortality rates in our two cohorts (Swiss: 20 and 15\% in Germany) are comparable to other studies on severely injured trauma patients $[16,32,33]$. Therefore, identified specific characteristics and differences between the two trauma systems by this investigation might also be interesting for other non-German-speaking countries, especially as 
a recent study showed that organization of EMS systems in Europe continues to vary largely from each other [34]. Given the specific conditions of both well-developed countries, it is impossible to extrapolate the findings of this study to less well-developed trauma systems/regions.

The key limitation of this study is that analysis was performed retrospectively, although data was collected prospectively. Additionally, only 3 out of 12 Swiss trauma institutes were included in the database.

Given the large sample size of both cohorts, comparing both countries by performing regular statistical testing will lead to many clinically irrelevant statistical differences. Therefore, we decided not to include these calculations in our report, and multifactorial analysis on mortality was performed by a comparison of RISC-II scoring. Furthermore, mortality rates might have been slightly affected by differences in local and cultural standards regarding treatment withdrawal of life support in specific cases such as neurotrauma.

Besides, another limitation of the study is the absence of relevant data on patients' admission and ICU respiratory status (such as ventilator settings, $\mathrm{pCO} 2, \mathrm{pO} 2$ values). This made the interpretation of encountered differences in intubation/chest tube placement rates more complicated and should be focus of future studies.

In contrast to most other comparative international studies on trauma care, the current study is unique as it is based on uniform data collecting protocols for both groups (ensured by the utilization of standardized in TraumaRegister DGU ${ }^{\circledR}$ forms). As inclusion criteria were similar for both cohorts as well, standardization increased and selection bias issues were avoided as much as possible.

Standardization in polytrauma studies has always been an important and difficult issue. Due to the large number of included cases, variability was minimized, and standardization was optimized in our study [35]. Given the utilization of similar in-hospital treatment algorithms for trauma patients (Advanced Trauma Life Support [36] and S3-Trauma guidelines [10]) in both countries and documentation in the same native language, standardization further gained.

\section{Conclusion}

The current study identified differences in pre-hospital care routines and outcome between two German-speaking countries in Western Europe: Switzerland and Germany. Despite organizational and operational differences in pre-hospital trauma care, standardized mortality rates were similar. Slightly higher crude mortality in Switzerland may be associated with less emergency doctor presence on the scene, even in a well renowned pre-clinical trauma system with highly qualified paramedics.
Therefore, further analysis of specific subgroups of trauma patients and benchmarking studies are indicated to optimize patient care and utilization of resources. Based on our findings, we suggest focusing on pre-hospital medical physician involvement intubation and fluid administration necessity in Germany as well as on geriatric and non-rural trauma care and the prevention of violence-related trauma in Switzerland.

\section{Compliance with ethical standards}

Conflict of interest The authors declare that they have no conflict of interest. RL declares that his institution receives an ongoing support from AUC GmbH which is the owner of the registry data. This service agreement includes statistical support for scientific publications using registry data.

Compliance with ethical requirements This study has been performed in accordance with the ethical standards laid down in the 1964 Declaration of Helsinki and its later amendments. Due to this ethical approval no consent to participate in the study was needed, because only anonymized data were used.

\section{References}

1. Ali J, Adam RU, Gana TJ, Williams JI. Trauma patient outcome after prehospital trauma life support program. J Trauma. 1997;42(6):1018-21.

2. van Olden GD, Meeuwis JD, Bolhuis HW, Boxma H, Goris RJ. Clinical impact of advanced trauma life support. Am J Emerg Med. 2004;22(7):522-5

3. Nathens AB, Jurkovic GJ, Rivara FP, Maier RV. Effectiveness of state trauma systems in reducing injury-related mortality: a national evaluation. J Trauma. 2000;48:25-30.

4. Celso B, Tepas J, Langland-Orban B, Pracht E, Papa L, Lottenberg L, et al. A systematic review and meta-analysis comparing outcome of severely injured patients treated in trauma centers following the establishment of trauma systems. J Trauma. 2006;60:371-8.

5. Westhoff J, Hildebrand F, Grotz M, Richter M, Pape HC, Krettek C. Trauma care in Germany. Injury. 2003;34(9):674-83.

6. Allgoewer M. Trauma systems in Europe. Am J Surg. 1991;161(2):226-9.

7. Garnowski M, Manner H. On factors related to car accidents on German Autobahn connectors. Accid Anal Prev. 2011;43(5):1864-71.

8. European Commission, Directorate General for Transport. European Commission, Road Safety Country Overview-Germany/ Switzerland, September 2016.

9. Rossi R. Early care or quick transport? The effectiveness of preclinical treatment of emergency patients. Anaesthesist. 1997;46(2):126-32.

10. Polytrauma Guideline Update Group. Level 3 guideline on the treatment of patients with severe/multiple injuries. Eur J Trauma Emerg Surg. 2018;44(Suppl 1):3-271.

11. Jones A, Donald MJ, Jansen JO. Evaluation of the provision of helicopter emergency medical services in Europe. Emerg Med J. 2018;35(12):720-5

12. Pittet V, Burnand B, Yersin B, Carron PN. Trends of pre-hospital emergency medical services activity over 10 years: a populationbased registry analysis. BMC Health Serv Res. 2014;14:380. 
13. Kleber C, Giesecke MT, Tsokos M, Haas NP, Schaser KD, Buschmann CT. Overall distribution of trauma-related deaths in Berlin 2010: advancement or stagnation of German trauma management? World J Surg. 2012;36:2125-30.

14. Taylor P. Standardized mortality ratios. Int J Epidemiol. 2013;42(6):1882-900.

15. Staiger RD, Schwandt H, Puhan MA, Clavien PA. Improving surgical outcomes through benchmarking. Br J Surg. 2019;106(1):59-64.

16. Haider AH, Hashmi ZG, Gupta S, et al. Benchmarking of trauma care worldwide: the potential value of an international trauma databank (ITDB). World J Surg. 2014;38:1882-911.

17. Demetriades D, Murray J, Sinz B, Myles D, Chan L, Sathyaragiswaran L, et al. Epidemiology of major trauma and trauma deaths in Los Angeles County. J Am Coll Surg. 1998;187(4):373-83.

18. Kehoe A, Smith JE, Edwards A, Yares D, Lecky F. The changing face of major trauma in the UK. Emerg Med J. 2015;32(12):911-5.

19. Christensen MC, Ridley S, Lecky FE, Munro V, Morris S. Outcomes and costs of blunt trauma in England and Wales. Crit Care. 2008;12:R23.

20. Nathens AB et al. ACS Committee on Trauma. NTDB Annual Report 2012: https://www.facs.org/trauma/ntdb/ntdbannualrepor t2010.pdf

21. Crewdson K, Lockey DJ, Roislien J, Lossius HM, Rehn M. The success of pre-hospital tracheal intubaton by different pre-hospital providers: a systematic literature review and meta-analysis. Crit Care. 2017;21(1):31.

22. Gunkel S, Konig M, Albrecht R, Bruesch M, Lefering R, Sprengel K, Werner CM, Simmen HP, Wanner GA. Status quo der Boden- und Luftrettung schwerverletzter patienten: analyse eines uberregionalen Schweizer traumazentrums. Unfallchirurg. 2015;118(3):233-9. https://doi.org/10.1007/s00113-014-2567-6.

23. Brinck T, Raj R, Skrifvars MB, Kivisaari R, Siironen J, Lefering R, Handolin L. Unconscious trauma patients: outcome differences between southern Finland and Germany-lesson learned from trauma-registry comparisons. Eur J Trauma Emerg Surg. 2016;42(4):445-51. https://doi.org/10.1007/s00068-015-0551-7 (Epub 2015 Jul 21).

24. Kaserer A, Stein P, Simmen HP, Spahn DR, Neuhaus V. Failure rate of prehospital chest decompression after severe thoracic trauma. Am J Emerg Med. 2017;35(3):469-74.

25. Timm A, Maegele M, Lefering R, Wendt K, Wyen H, the TraumaRegister DGU. Pre-hospital rescue times and actions in severe trauma. A comparison between two trauma systems: Germany and the Netherlands. Injury. 2014;455:543-52.
26. Kudo D, Yoshida Y, Kushimoto S. Permissive hypotension/ hypotensive resuscitation and restricted/controlled resuscitation in patients with severe trauma. J Intensive Care. 2017;5:11.

27. Driessen A, Froehlich M, Schaefer N, Mutschler M, Defosse JM, Brockamp T, et al. Prehospital volume resuscitation: did evidence defeat the crystalloid dogma? An analysis of the TraumaRegister DGU ${ }^{\circledR}$ 2002-2012. Scand J Trauma Resusc Emerg Med. 2016;24:42.

28. Stein P, Kaserer A, Sprengel K, Wanner GA, Seifert B, Theusinger OM, Spahn DR. Change of transfusion and treatment paradigm in major trauma patients. Anaesthesia. 2017;72:1317-26. https:// doi.org/10.1111/anae.13920.

29. Kaserer A, Casutt M, Sprengel K, Seifert B, Spahn DR, Stein P. Comparison of two different coagulation algorithms on the use of allogenic blood products and coagulation factors in severely injured trauma patients: a retrospective, multicentre, observational study. Scand J Trauma Resusc Emerg Med 2018;26(4):4. https:// doi.org/10.1186/s13049-017-0463-0.

30. Bieler D, Franke A, Lefering R, Hentsch S, Willms A, Lulla M, et al. Does the presence of an emergency physician influence pre-hospital time, pre-hospital interventions and the mortality of severely injured patients? A matched-pair analysis based on the trauma registry of the German Trauma Society (TraumaRegister DGU $^{\circledR}$ ). Injury. 2017;48(1):32-40.

31. Osterwalder JJ. Mortality of blunt polytrauma: a comparison between emergency physicians and emergency medical technicians-prospective cohort study at a level I hospital in eastern Switzerland. J Trauma. 2003;55(2):355-61.

32. Brinck T, Handolin L, Paffrath T, Lefering R. Trauma registry comparison: 6-year results in trauma care in Southern Finland and Germany. Eur J Trauma Emerg Surg. 2015;41:509-16.

33. Spicer R, Miller T, Langley J, et al. Comparison of injury case fatality rates in the United States and New Zealand. Inj Prev. 2005;11:1-76.

34. Seblova J, Cimpoesu D, Khoury A, Revue E, Trenkler S. Prehospital emergency care systems in Europe-EuSEM prehospital sectionsurvey 2016. Eur J Emerg Med. 2018;25(6):446-7.

35. Gundersen HJ, Osterby R. Optimizing sampling efficiency of stereological studies in biology: or 'do more less well!'. J Microsc. 1981;121:63-73.

36. ATLS Subcommittee, American College of Surgeon Committee on Trauma, International ATLS Working Group. Advanced trauma life support $\left(\right.$ ATLS $\left.^{\circledR}\right)$ : the ninth edition. J Trauma. 2013;74(5):1363-6 